\title{
Numerical Thermal Analysis of a T Jump System Used for Studying Polymer Behaviour
}

\author{
S. Gomes ${ }^{1, a}$, P. Pascoal-Faria ${ }^{1,2, b^{*}}$, G.R. Mitchell ${ }^{1, c}$, T. Gkourmpis ${ }^{3, d}$ \\ and T.Youngs ${ }^{4, e}$ \\ ${ }^{1}$ Centre for Rapid and sustainable Product Development, Institute Polytechnic of Leiria \\ ${ }^{2}$ Department of Mathematics, School of Technology and Management, Polytechnic Institute of \\ Leiria, Rua General Norton de Matos, Apartado 4133, 2411-901 Leiria - Portugal \\ ${ }^{3}$ Department of Materials \& Manufacturing Technology, Chalmers University of Technology, \\ Gothenburg SE-412 93, Sweden \\ ${ }^{4}$ ISIS Neutron and Muon Source, STFC Rutherford Appleton Laboratory, Harwell Campus, Didcot, \\ OX11 0QX, United Kingdom

\begin{abstract}
asara.gomes@ipleiria.pt, bpaula.faria@ipleiria.pt, 'cgeoffrey.mitchell@ipleiria.pt, dthomas.gkourmpis@chalmers.se, etristan.youngs@stfc.ac.uk
\end{abstract}

Keywords: thermal analysis, polymer, finite element method; crystallisation

\begin{abstract}
The processing of polymers is highly complex. The study of their crystallisation assumes an important role and needs to be carefully detailed. Scattering experiments can be used to study polymer molecular organisation. However these procedures are still very multifaceted leading to the need for planning all the details in the experiments that are to be performed. This manuscript presents a finite element model developed to study the temperature variation of a T Jump System, which has been used for studying polymer behaviour with the NIMROD instrument at the ISIS Neutron and Muon Source, UK. Results show that the variation across the sample was $2^{\circ} \mathrm{C}$ at a maximum temperature of $70^{\circ} \mathrm{C}$ and $1{ }^{\circ} \mathrm{C}$ at a maximum temperature of $50^{\circ} \mathrm{C}$.
\end{abstract}

\section{Introduction}

Polymers are used worldwide in many different applications. The study of polymer crystallisation is paramount for better understanding of their behaviour. It has been studied since the experiments of Keller [1], in which he discovered the formation of chain-folded lamellar crystals. The crystallisation of polymers occurs when its chains evolve from random to ordered configurations [2][3]. It involves a conformational change, so the segments of chain with regular conformation can be obtained with different length scales, such as: i) the folded lamellar thickness of $\sim 10 \mathrm{~nm}$, ii) the crystal unit cell of $\sim 1 \mathrm{~nm}$; and iii) the detail of the chain formation of $\sim 0.1 \mathrm{~nm}$. These different length scales are usually addressed separately using different instrumentation and techniques [2].

Scattering experiments are an important technique when evaluating the molecular organisation of polymers. Neutron scattering, applied in this work, uses neutrons produced in nuclear reactors or via a spallation source which scatter from the atomic nuclei within the sample, with the momentum transfer of the scattered neutrons providing structural information on the sample [2]. This technique allows the study of the rearrangement of molecules, including the conformational rearrangements that occur before crystallisation. The results show the range of data intrinsic for a spallation source operating in time-of-flight mode to generate a single data set, as illustrated in Figure 1 when obtained from NIMROD [4]. The presented illustration shows a differential scattering cross section as obtained from NIMROD's experimental setup ranging from $Q=0.02$ to $50 \AA^{-1}$ and is on a single vertical scale in absolute units. This data contains a wide range of correlations spaning from the local chain conformation to the spatial arrangement of chain segments [5][6]. Figure 1 shows data for a poly ( $\varepsilon$-caprolactone) sample at $20^{\circ} \mathrm{C}$ in the semi-crystalline state and at $80^{\circ} \mathrm{C}$ in the melt state [4]. These differential scattering cross-section data were obtained using the Near and InterMediate Range Order Diffractometer (NIMROD) at the ISIS Neutron and Muon Source, located at the STFC 
Rutherford Appleton Laboratory in the United Kingdom [2][7]. The data obtained from NIMROD allows the development of exhaustive and well constrained models of complex scattering systems, and cover the three critical length scales in the ordering process [8][9].

One of the experimental approaches considered for the study of the polymers transformations using NIMROD consists in rapidly cooling the sample from the melt state to a temperature lower than the melting point. One of the main challenges of this work is the need to cool the sample fast enough to prevent the transformation to the more ordered state [9]. Such study is paramount in allowing better control and understanding of the crystallisation process. Therefore, this paper presents a numerical thermal analysis of a T Jump System used to control the temperature of the sample, and which was adopted for the experiments performed with NIMROD.

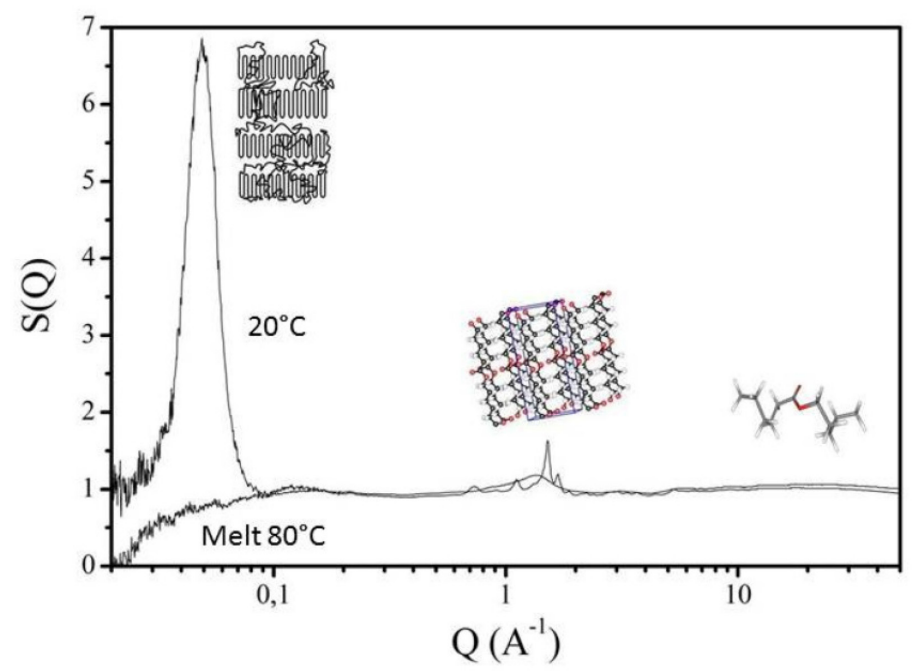

Figure 1: Differential scattering cross section of a deuterated poly( $\varepsilon$-caprolactone) obtained at NIMROD at $20^{\circ} \mathrm{C}$ in the semi-crystalline state and at $80^{\circ} \mathrm{C}$ in the melt state [4].

\section{T Jump System}

In order to overcome the possibilities of undercooling the sample past the selected crystallisation temperature, the T Jump System shown in Figure 2 was developed and tested at NIMROD [4][8]. The system uses a circular scheme for cooling or heating the sample with ethylene glycol and water, using two temperatures alternators, both set at two different temperatures, which circulates through the copper block with a flow rate between 22 to $26 \mathrm{~L}$ per minute [8]. The circulation system is controlled using a microcontroller so the valves that regulate the high and low temperatures current can be switched automatically [4]. It is composed of a copper mould with two vanadium sheets holding on the polymer sample of polycaprolactone (PCL) in the middle. The T Jump System was designed to use a large area sample to allow for the maximum area of illumination by the neutron beam, and to ensure that undercooling of the sample past the crystallisation temperature can be avoided. In the NIMROD instrument the sample stage area is evacuated (in vacuum). Results showed that the system was efficacious in cooling the sample to the crystallisation temperature when any crystallisation at higher temperatures [4][8]. 


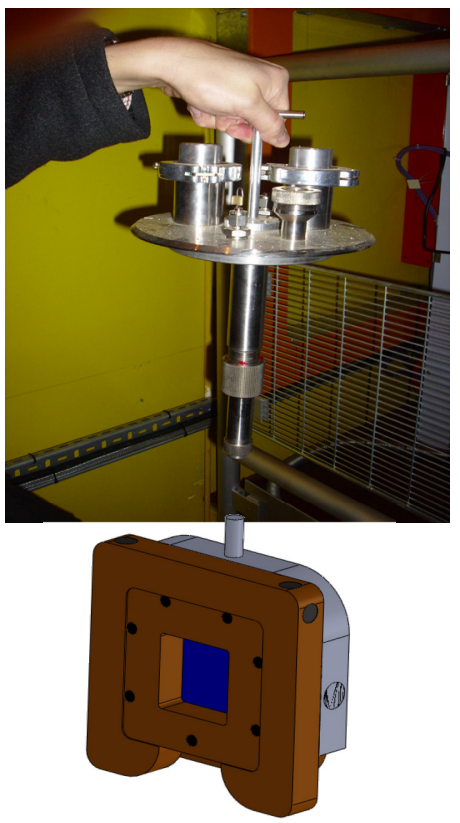

(a)

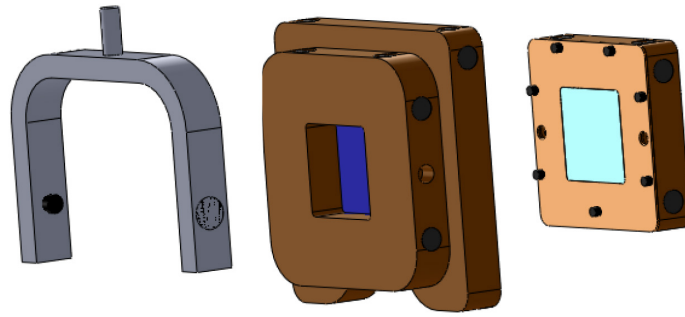

(b)

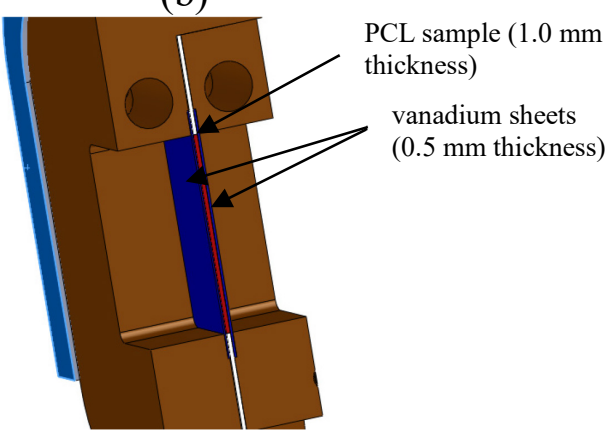

(c)

Figure 2: T Jump system: (a) overview of the support structure and the system, (b) copper blocks; and (c) internal structure of the copper blocks, that includes 2 vanadium sheets and a PCL sample.

\section{Numerical Model}

A transient numerical thermal analysis using a finite element model to determine the temperature distribution over time was performed. The heat transfer was considered to occur by radiation from the copper mould to the environment, and by conduction between the different parts. Moreover, no convection was considered since the system was placed in vacuo. Materials were considered to have an isotropic behaviour, and the thermal conductivity for each material was assumed according to the values presented in Table 1. An environmental temperature of $22^{\circ} \mathrm{C}$ was employed.

Table 1 - Thermal conductivity of materials.

\begin{tabular}{ccc}
\hline Material & $\begin{array}{c}\text { Thermal conductivity } \\
\left(\mathrm{W} . \mathrm{m}^{-1} \mathrm{~K}^{-1}\right)\end{array}$ & $\begin{array}{c}\text { Density } \\
\left(\mathrm{kg}^{-3}\right)\end{array}$ \\
\hline Copper & 385 & 7764 \\
\hline PCL & 0.128 & 1160 \\
\hline Vanadium & 31 & 6110 \\
\hline
\end{tabular}

A three-dimensional model was implemented using finite elements with: i) 10 nodes tetrahedron for the copper mould; and ii) 20 nodes hexahedron ("brick" element) for the vanadium sheets and the PCL sample, in a total of 357683 elements (see Figure 3). A perfect bond between all elements was assumed. The heating channels were simulated through the consideration of temperature at the channels walls, as illustrated in Figure 4. The temperature was applied according to the scheme shown in Figure 5, where a total time of $200 \mathrm{~s}$ was taken into account to simulate the sudden temperature change from $70^{\circ} \mathrm{C}$ to $50^{\circ} \mathrm{C}$ at $100 \mathrm{~s}$. Also the heating of the sample from the environment temperature to $70^{\circ} \mathrm{C}$ was considered. 


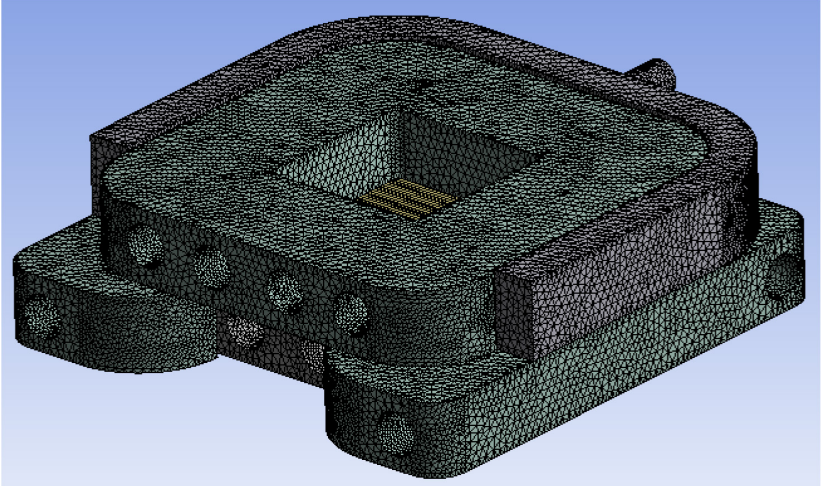

(a)

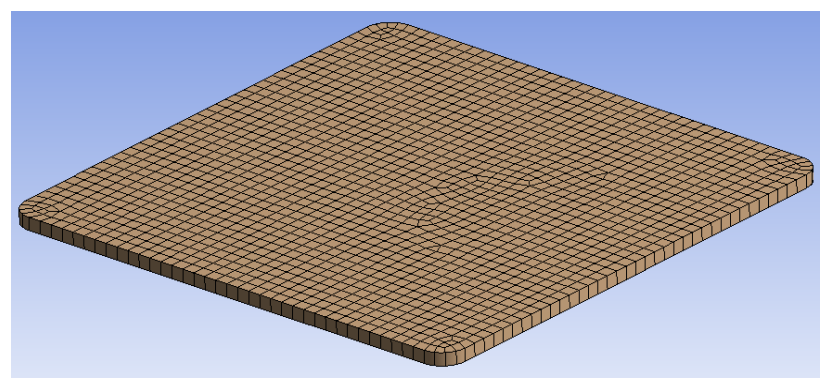

(b)

Figure 3: Model mesh: (a) model overview; and (b) PCL sample.

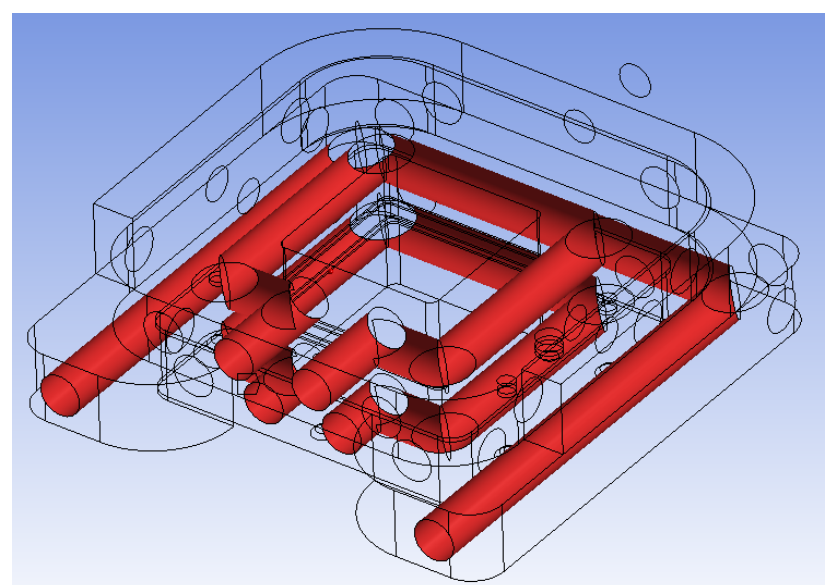

Figure 4: Temperature application at the channels.

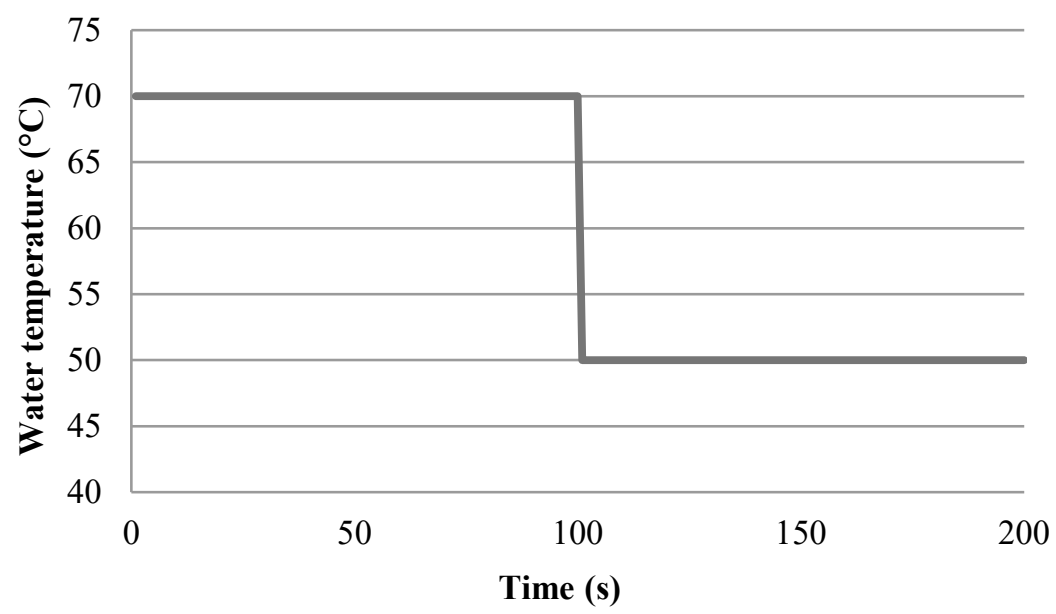

Figure 5: Input water temperature variation over time.

\section{Results}

The numerical analysis allowed the evaluation of the temperature stabilisation after the change. The temperature distribution on the copper mould and sample at $75 \mathrm{~s}$ and $200 \mathrm{~s}$ are shown in Figures 6 (a) to (d). The maximum temperatures determined at the copper mould were $70^{\circ} \mathrm{C}$ at $75 \mathrm{~s}$ and $50^{\circ} \mathrm{C}$ at $200 \mathrm{~s}$. The maximum temperature was verified to occur at the copper mould (see Figures 6 (a) and (b)), which varies less than $0.1{ }^{\circ} \mathrm{C}$ (see Figures $6(\mathrm{c})$ and (d)). On the other hand, the minimum temperature was seen to occur at the vanadium sheets and the PCL sample, achieving 2 and $1{ }^{\circ} \mathrm{C}$ lower than that verified in the copper mould (see Figures $6(\mathrm{a})$ and (b)). The movement of 
the temperature from the copper mould to the sample occurs by conduction, leading the sample edges get the same temperature as the copper mould.

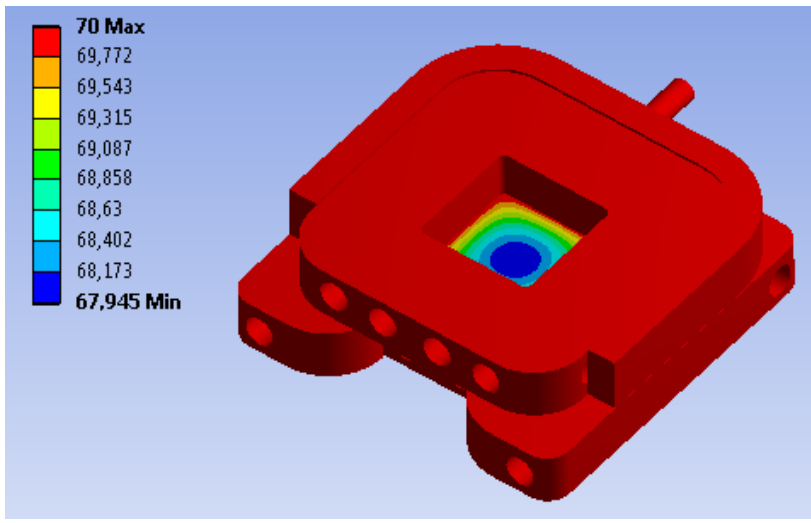

(a)

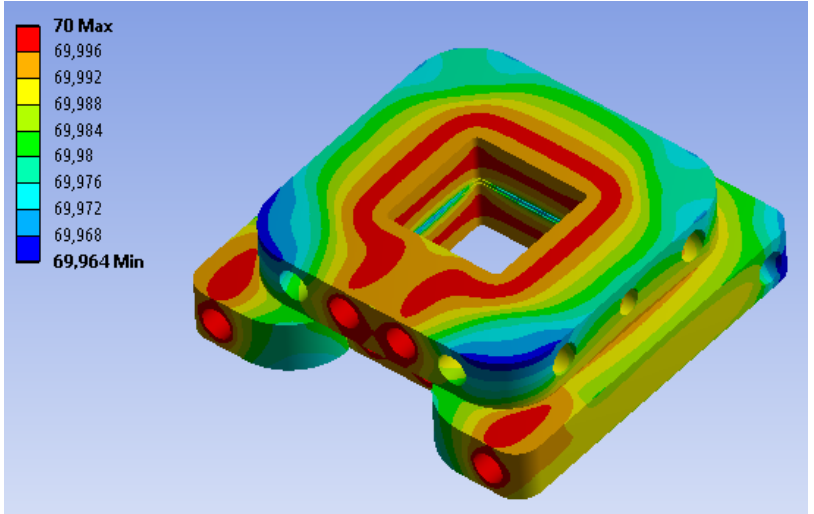

(c)

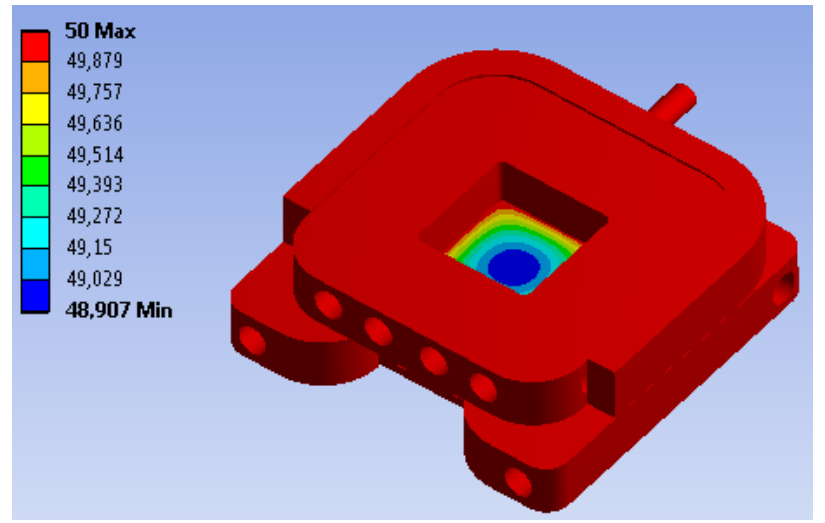

(b)

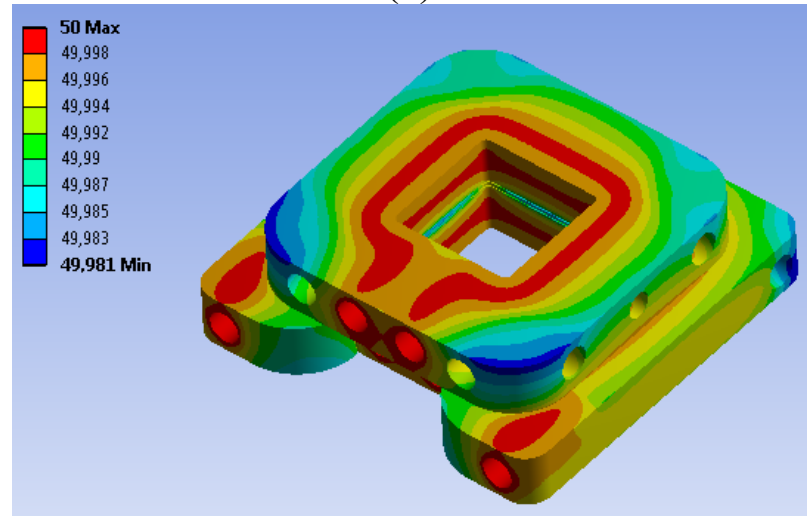

(d)

Figure 6: Temperature variation of the T Jump System in ${ }^{\circ} \mathrm{C}$ : (a) overall view at $t=75 \mathrm{~s}$, (b) overall view at $\mathrm{t}=200 \mathrm{~s},(\mathrm{c})$ copper mould at $\mathrm{t}=75 \mathrm{~s}$; and (d) copper mould at $\mathrm{t}=200 \mathrm{~s}$.

The temperature calculated at the sample when the time was equal to $75 \mathrm{~s}$ and $200 \mathrm{~s}$ is illustrated in Figures 7 (a) and (b). As already stated, the interior sample area does not reach the same temperature as the copper. Figure 8 illustrates the distribution of the temperature across the sample. As it is possible to see, temperature variation is at maximum $2^{\circ} \mathrm{C}$ in $20 \mathrm{~mm}$ at $75 \mathrm{~s}$; and $1^{\circ} \mathrm{C}$ in $20 \mathrm{~mm}$ at $200^{\circ} \mathrm{C}$. These results are positive since one of the objectives of the T Jump System was to reduce the temperature differences over the sample, and therefore it was accomplished. The temperature variation can be reduced by increasing the thickness of the vanadium sheets, but since the differences are already very small this was not experimentally performed [8].

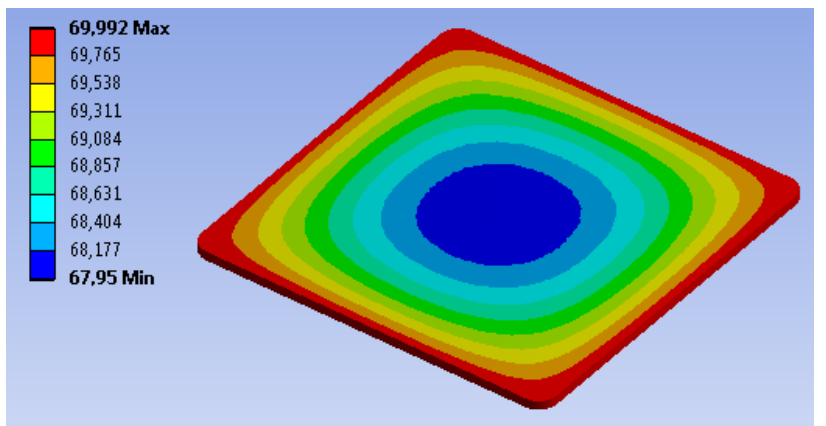

(a)

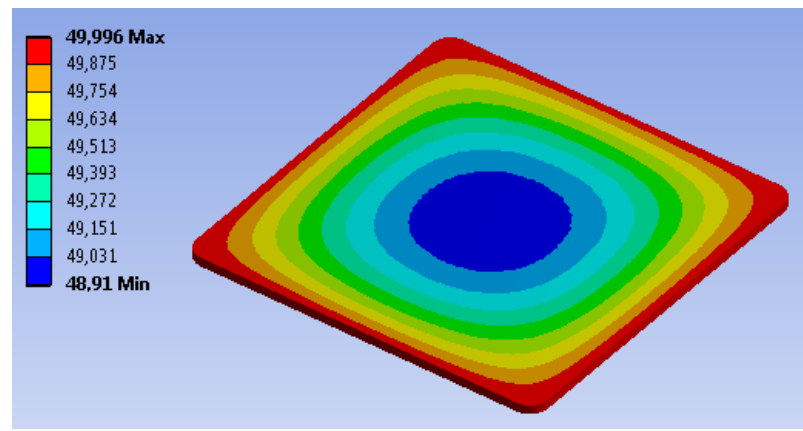

(b)

Figure 7: Temperature variation on the sample in ${ }^{\circ} \mathrm{C}$ : (a) $\mathrm{t}=75 \mathrm{~s}$; and (b) $\mathrm{t}=200 \mathrm{~s}$. 


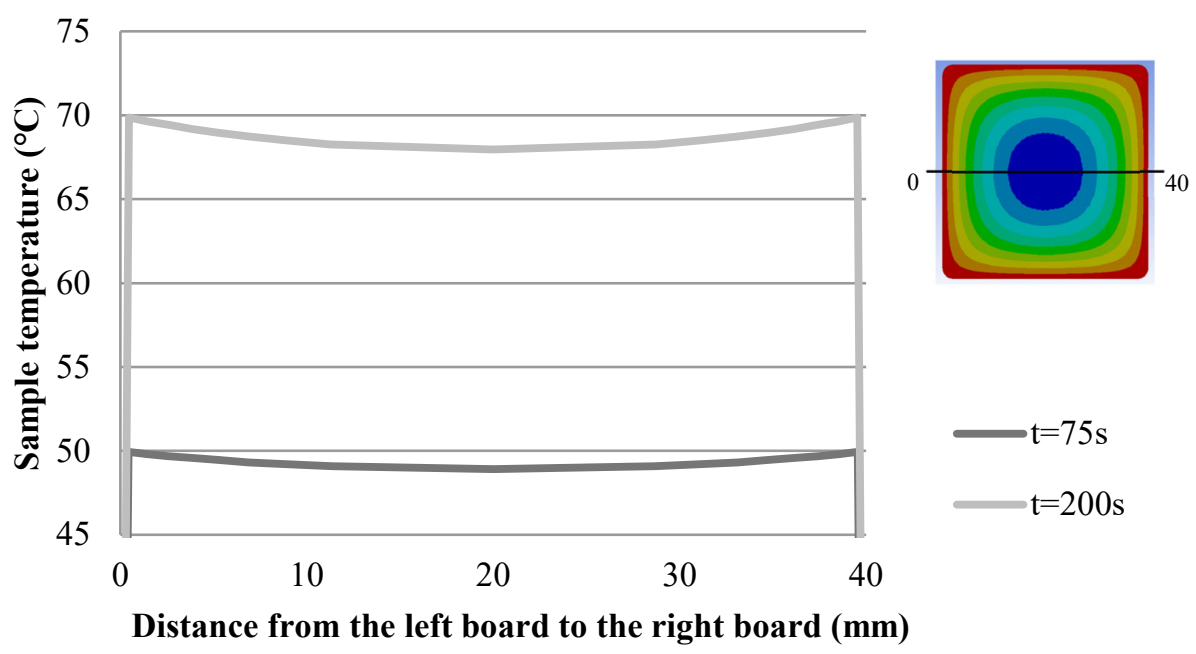

Figure 8: Temperature distribution of the sample cross the section at $t=75 \mathrm{~s}$ and $\mathrm{t}=200 \mathrm{~s}$.

The sample maximum and minimum temperatures over the analysis are shown in Figure 9. It is possible to verify that after the temperature reduction, its stabilisation occurs during a period of $2 \mathrm{~s}$. This is given to the fact that, besides copper being a good conductor of heat, the vanadium sheet is in contact with the sample and the sample itself is relatively thin in comparison $(0.5 \mathrm{~mm}$ and $1.0 \mathrm{~mm}$ ), and therefore the thermal transfer occurs rapidly. Moreover, it is important to highlight that this analysis did not take into account the time consumed during the liquids change, which can influence the computed stabilisation period. Additionally, as shown in Figure 9, the sample presents a temperature lower than $50^{\circ} \mathrm{C}$, whose is constant until the end of the analysis, and showing that the temperature at the sample can be controlled to avoid undercooling the sample past the selected crystallisation temperature.

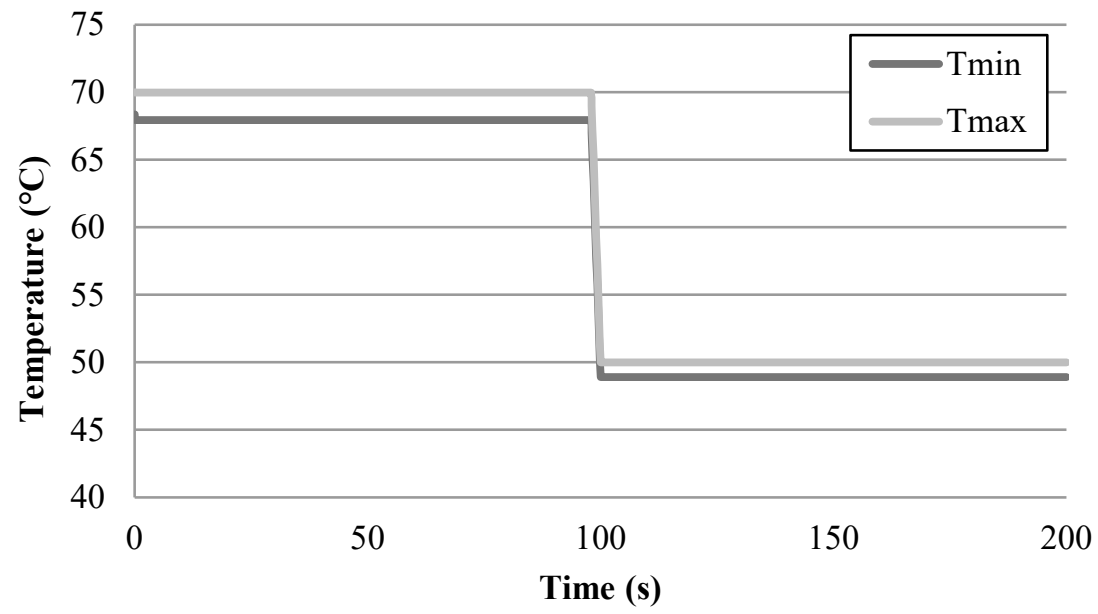

Figure 9: Minimum and maximum temperatures variation at the sample over the analysis.

\section{Conclusions}

A numerical thermal analysis was performed for studying the temperature distribution of a T Jump System employed during scattering experiments performed on NIMROD. Results showed that temperature stabilisation of the sample occurs rapidly, and that the temperature at its centre reduces to values lower than the water minimum temperature. However, since this value remains constant, the temperature at the sample can be controlled in order to avoid undercooling the sample past the crystallisation temperature, when the T Jump System is used. 
The temperature variation over the sample area is at maximum $2^{\circ} \mathrm{C}$ in $20 \mathrm{~mm}$ at $75 \mathrm{~s}$ and $1{ }^{\circ} \mathrm{C}$ in $20 \mathrm{~mm}$ at $200 \mathrm{~s}$, which is positive since it was pretended to achieve an equal temperature distribution over the analysis. It also shows that the T Jump System is a correct device to be used in this type of experiments, and that an incensement of the vanadium sheets thickness can benefit the uniformity of temperature.

Several improvements are expected to be implemented in the near future to improve the model, including a fluid dynamic analysis to consider the time consumed during the liquid change in the channels.

\section{Acknowledgements}

The authors would like to acknowledge the financial support from Fundação da Ciência e Tecnologia (Portugal) as part of the UC4EP (Understanding Crystallisation for Enhanced Polymer Properties) at CDRSP PTDC/CTM-POL/7133/2014, UID/Multi/04044/2013, PAMI No 022158 and the Fundo Europeu de Desenvolvimento Regional (FEDER), Programa Portugal 2020, through the Programa Operacional Regional do Centro 2020 (CENTRO-01-0145-FEDER-022158). The neutron scattering measurement was performed at the STFC ISIS facilities (United Kingdom).

\section{References}

[1] Keller, A., A note on single crystal in polymers - evidence for a folded chain configuration, Philosophical Magazine, 2(21):1171 (1957).

[2] G. R. Mitchell, A. Tojeira, Controlling the Morphology of Polymers: Multiple Scales of Structures and Processing, Springer (2017).

[3] G. R. Mitchell, Order in the Amorphous State of Polymers, S. E. Keinath, R. L. Miller, J. K. Rieke, Eds., Plenum: New York (1978).

[4] G. R. Mitchell, A. Mateus, A. Tojeira, D. Bowron, T. Gkourmpis, S. Mohan, F. Davis, Experimental Approach to the study of multiscale structural development polymers, 5th International Conference on Polymer Behavior, Aveiro Portugal (2012).

[5] G. Strobl, Colloquium: Laws controlling crystallization and melting in bulk polymers, Reviews of Moderns Physics, 81, 1287 (2009).

[6] G. R. Mitchell, Neutron Diffraction to Soft Matter in Essentials of Neutron Techniques for Soft, Eds Imae, Toyoko et al. (2011).

[7] D. T. Bowron, A. K. Soper, K. Jones, S. Ansell, S. Birch, J. Norris, L. Perrot, D. Riedel, J. Rhodes, A. Botti, M. A. Ricci, F. Grazzi, M. Zoppi, NIMROD: the Near and InterMediate Range of the ISIS second target station, Review of Scientific Instruments, 81, 033905 (2010).

[8] G. R. Mitchell, A. Mateus, F. Davis, S. Mohan, T. Gkourmpis, T. Youngs, New Tools for understanding Complex Polymer Behaviour, Procedia Manufacturing, (2017) 12, 280-290

[9] G. R. Mitchell, D. Bowron, A. Mateus, P. Bartolo, T. Gkourmpis, K. Phomphrai, D. Lopez, F. Davis, SANS/WANS time-resolving neutron scattering studies of polymer phase transitions using NIMROD, MRS Proceedings, 1528, 83-88 (2013). 